\title{
MiR-452 negatively regulates osteoblast differentiation in periodontal ligament stem cells by targeting the polycomb- group protein, BMI1
}

\author{
Tiantian Mao, Jun Li, Ruobing Peng, Linhua Liu, Youjian Peng* \\ Department of Stomatology, Renmin Hospital of Wuhan University, Wuhan, Hubei Province 430060, China
}

*For correspondence: Email: yj_peng66@163.com; Tel: +86-027-88041911

Sent for review: 19 November 2020

Revised accepted: 26 March 2021

\begin{abstract}
Purpose: To determine whether miR-452 regulates osteoblast differentiation (OD) in human periodontal ligament stem cells (hPDLSCs) by targeting polycomb-group protein BMI1.

Methods: hPDLSCs were stimulated to differentiate upon treatment with mineralization liquid. Quantitative real-time polymerase chain reaction ( $q R T-P C R$ ) and western blotting were used to measure mRNA and protein expressions, respectively. Alkaline phosphatase (ALP) activity and Alizarin red staining were used to determine the osteogenic differentiation (OD) of $h P D L S C s$. The bioinformatics software, Targetscan, was used to predict the potential target of miR-452, while luciferase assay, qRT$P C R$, and western blot were employed to verify the target gene of miR-452, BMI1.

Results: MiR-452 was downregulated during the OD of hPDLSCs, but miR-452 overexpression inhibited the OD of hPDLSCs. BMI1 was identified as a direct target gene of miR-452 during the OD of hPDLSCS, while miR-452 overexpression correlated inversely with BMI1 expression during OD of hPDLSCS.

Conclusion: Overexpression of miR-452 suppresses the OD of hPDLSCs by targeting BMI1.This study may provide potential diagnostic and therapeutic basis for OD in hPDLSCs.
\end{abstract}

Keywords: MiR-452, Osteogenic differentiation, Periodontal ligament stem cells, BMI1

\begin{abstract}
This is an Open Access article that uses a fund-ing model which does not charge readers or their institutions for access and distributed under the terms of the Creative Commons Attribution License (http://creativecommons.org/licenses/by/4.0) and the Budapest Open Access Initiative (http://www.budapestopenaccessinitiative.org/read), which permit unrestricted use, distribution, and reproduction in any medium, provided the original work is properly credited.

Tropical Journal of Pharmaceutical Research is indexed by Science Citation Index (SciSearch), Scopus, International Pharmaceutical Abstract, Chemical Abstracts, Embase, Index Copernicus, EBSCO, African Index Medicus, JournalSeek, Journal Citation Reports/Science Edition, Directory of Open Access Journals (DOAJ), African Journal Online, Bioline International, Open-J-Gate and Pharmacy Abstracts
\end{abstract}

\section{INTRODUCTION}

Periodontal tissue damage causes oral organ defects and has adverse effects on health for many people [1]. Human periodontal ligament stem cells (hPDLSCs) from periodontal ligament tissue can form new periodontal supporting tissues and exert effects on the functional regeneration and biological repair of periodontal tissue [2]. MiRNAs are classified of short noncoding RNAs (18-25 nucleotides), which function as gene suppressors to downregulate the stability or translational efficiency of target genes by the 3'-untranslated region (3'-UTR), and can regulate cell proliferation, migration, and differentiation [3,4].

Numerous studies have revealed the key roles of miRNAs in osteogenic differentiation (OD) of stem cells. For instance, in bone marrow 
mesenchymal stem cells (BMMSCs), miR-204 promotes adipogenic differentiation by regulating expression of Runx2 and thereby inhibiting OD [5], whereas miR-26a promotes OD of adiposederived mesenchymal stem cells (ADMSCs) [6]. This study showed that miR-452 expression was downregulated during OD in PDLSCs, indicating that miR-452 could play an important role in the progression of OD.

B cell-specific Moloney murine leukemia virus integration site 1 (BMI1) is a key component of the polycomb repressive complex 1 . It has been shown that knockdown of BMI1 could reduce alveolar bone mass in mice [7]. However, whether the binding interaction between BMI1 and miR-452 could play a role in the pathological process of OD remains unknown. Therefore, this experimental study aimed to determine the role of miR-452 in the progression of OD in PDLSCs, and explore the underlying mechanism of action.

\section{EXPERIMENTAL}

\section{Reagents}

Radio-immunoprecipitation assay (RIPA) buffer, Revert Aid RT Reverse Transcription kit, antibodies against Runx2, osterix, OCN, BMI1, and $\beta$-actin, Lipofectamine 2000, the Alkaline phosphatase (ALP) activity kit were purchased from Thermo Fisher Scientific (Waltham, MA, USA). Cell culture materials were from Gibco Life Technologies (Grand Island, NY, USA). The primers, BMI1-3'UTR wild type (BMI1 3'UTR-WT) and BMI1-3'UTR mutant (BMI1 3'UTR-MUT) were purchased from Sangon Biotech Company (Shanghai, China). Total RNA was extracted using the RNeasy Mini Kit (QIAGEN GmbH, Hilden, Germany). The mRNA expression was quantified using the SYBR-Green RT-PCR kit (Takara Bio, Inc., Otsu, Japan). Locked nucleic acid (LNA)-miR-452 and LNA-miR-NC were from IBS Solutions (Shanghai, China). The miR-452 mimic, miR-452 inhibitor, negative controls (NC) of miR-452 mimic and inhibitor, and the short hairpin (shRNA) sequences targeting the BMI1 gene were synthesized by Shanghai Gene Pharma Corporation (Shanghai, China). Alizarin red and cell lysate buffer were purchased from Sigma-Aldrich (St Louis, MO, USA). The dual luciferase reporter assay system was purchased from Promega (Madison, WI, USA). ECL detection reagents were from Amersham (Tokyo, Japan).

\section{Cell culture}

hPDLSCs were isolated from 10 male patients (15-32 years old) who underwent molar extraction. The isolated hPDLSCs were cultured in a-MEM supplemented with FBS (10 \%), vitamin $C$ sodium salt $(50 \mu \mathrm{g} / \mathrm{mL}), \quad \beta$ glycerophosphate disodium salt hydrate (10 mmoL/L), dexamethasone $(0.1 \mu \mathrm{moL} / \mathrm{L})$, and penicillin/streptomycin $(100 \mathrm{U} / \mathrm{mL})$ in an incubator at $37^{\circ} \mathrm{C}$ under $5 \% \mathrm{CO}_{2}$.

\section{qRT-PCR assay}

Total RNA was extracted from cultured hPDLSCs using the RNeasy Mini Kit, and converted to cDNA using the Revert Aid RT Reverse Transcription kit. The mRNA expression was quantified by qRT-PCR using the SYBR-Green RT-PCR kit and GAPDH was used as a control. The primers were listed.

Table 1: qRT-PCR primers

\begin{tabular}{lll}
\hline $\begin{array}{l}\text { Gene } \\
\text { name }\end{array}$ & Forward (5'-3') & Reverse (5'-3') \\
\hline miR-452 & GCGAACTGTTTG & CAGTGCGTGTCG \\
& CAGAGG & TGGAGT \\
BMI1 & CAGTTAGGCAGT & GTTGTGGTGGAG \\
& ATGTAGTTTC & TGTAAGAGTGT \\
GAPDH & CCTGGCACCCAG & GCCGATCCACAC \\
& CACAAT & G GAGTACT \\
\hline
\end{tabular}

Each qRT-PCR reaction was performed in triplicate using three RNA samples. The relative expression was determined using the $2^{-\Delta \Delta C t}$ formula [8].

\section{Cell transfection}

The miR-452 mimic (miR-452), negative control (NC) of miR-452 mimic (miR-NC), miR-452 inhibitor (miR-452 inh), NC of inhibitor (miR-NC inh), and the short hairpin (shRNA) sequences targeting the BMI1 gene (5'-AAGGAGGAGGT GAATGATAAA-3'), LNA-miR-452 (50 nM) and LNA-miR-NC (50 nM) were transfected into hPDLSCs using Lipofectamine 2000.

\section{Alizarin red staining}

This assay was used to detect mineralization nodules in hPDLSCs. Briefly, hPDLSCs were washed (PBS), fixed with paraformaldehyde (4\% (w/v), $30 \mathrm{~min}$, at room temperature (RT)), stained with Alizarin red (30 $\mathrm{min}$, at RT), and washed (PBS) for three times. Staining was observed by microscopy.

\section{Measurement of ALP activity}

hPDLSCs were lysed with cell lysate buffer and then centrifuged (12,000 g, $10 \mathrm{~min})$. ALP activity was measured using the ALP activity kit. 


\section{Luciferase reporter assay}

The assay was used to evaluate the molecular interaction between $\mathrm{miR}-452$ and BMI1 in hPDLSCs. hPDLSCs were seeded into 24-well plates $\left(1.5 \times 10^{5}\right.$ cells/well), and were cotransfected with $30 \mathrm{nM}$ of miR-452 mimic or miR$\mathrm{NC}$ and fluorescent plasmid (300 ng of BMl1 3'UTR-WT or BMI1 3'UTR-MUT) for $24 \mathrm{~h}$ using Lipofectamine 2000 . The relative luciferase activity was determined.

\section{Western blot}

RIPA Buffer was used to lyse and extract total protein from hPDLSCs. Extracted protein was separated by SDS-PAGE, transferred to membranes, and blocked with milk (5\%). The membranes were incubated with anti-BMI1 (1:1000), anti- $\beta$-actin (1:2000), anti-Runx2 (1:2000), anti-osterix $(1: 1000)$, and anti-OCN $(1: 1000)$ antibodies (Thermo Fisher Scientific) at $4{ }^{\circ} \mathrm{C}$ overnight.

After washing with TBS-T solution 3-5 times, membranes were incubated with secondary antibody (1:5000 dilution) for $1 \mathrm{~h}$ at RT. ECL detection reagents were used to visualize the immune complexes. Image Pro software was used to calculate band intensities.

\section{Statistical analysis}

All experiments were repeated at least three times. The data are presented as mean \pm standard deviations (SD) and analyzed using SPSS 21.0 statistical software. ANOVA or $t$ test was used to assess significance $(p<0.05)$. The Dunnett multiple comparison test was applied to identify differences between groups.

\section{RESULTS}

\section{MiR-452 expression decreased in hPDLSCs during OD}

Based on whether hPDLSCs were stimulated to differentiate upon treatment with mineralization liquid, hPDLSCs were divided into OD group and control group. The miR-452 expression in hPDLSCs was lower on days 7 and 14 as compared with that at day0 ( $p<0.05$; Figure 1). However, the miR-452 expression in Control group showed no significant difference at day0, days 7 and 14 . These results indicated that the miR-452 expression was reduced in hPDLSCs during OD.

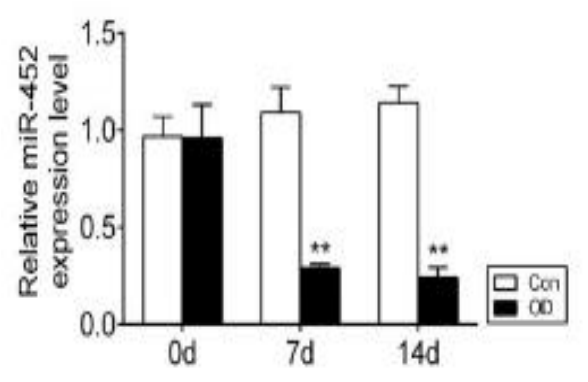

Figure 1. MiR-452 expression was decreased during OD of hPDLSCs; $\mathrm{n}=10,{ }^{* *} p<0.01$ vs. day 0

\section{MiR-452 overexpression inhibited OD of hPDLSCs}

To investigate the effects of miR-452 on OD of hPDLSCs, hPDLSCs were stably transfected with LV-miR-452. qRT-PCR showed that hPDLSCs transfected with LV-miR-452 expressed higher levels of miR-452 than that transfected with LV-NC ( $p<0.01$; Figure $2 \mathrm{~A}$ ), indicating the successful transfection. Compared with the control group, the ALP activity was increased in the OD group $(p<0.01)$. However, the induced ALP activity in OD group was inhibited by miR-452 overexpression ( $p<0.01$; Figure 2 B).

The results in Figure $2 \mathrm{C}$ showed that treatment with mineralization liquid promoted matrix mineralization of hPDLSCs in OD group $(p<$ 0.01). However, transfection with LV-miR-452 inhibited the progression of matrix mineralization of hPDLSCs in the OD group $(p<0.01)$. Consistently, the expression of OD markers, such as RUNX2, osterix, and OCN were upregulated in the OD group $(p<0.01)$, and transfection with LV-miR-452 reduced the expression of RUNX2, osterix, and OCN in the OD group $(p<0.01$; Figure $2 \mathrm{D})$. These results showed that miR-452 inhibits OD of hPDLSCs.

\section{BMI1 was the direct target of miR-452}

The bioinformatics software Targetscan (http://www.targetscan.org/vert_72/) revealed that BMI1 was predicted as a potential target of miR-452 (Figure $3 \mathrm{~A}$ ). The luciferase reporter assay was used to verify the binding affinity of miR-452 to the BMI1 3'UTR-WT in hPDLSCs. The luciferase activity in hPDLSCs that transfected with miR-452 mimic and BMI1 3'UTR-WT was decreased, but miR-452 mimics did not affect the luciferase activity in hPDLSCs that transfected with BMI1 3'UTR-MUT, indicating that BMI1-WT was a target of miR-452 (Figure $3 \mathrm{~B}$ ). 

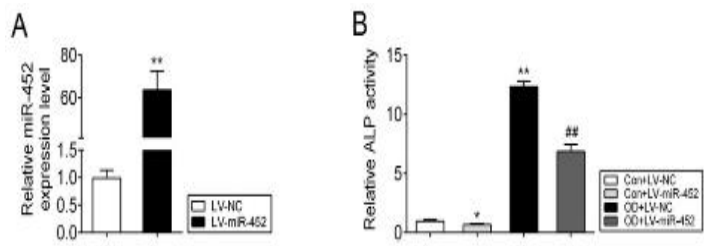

C

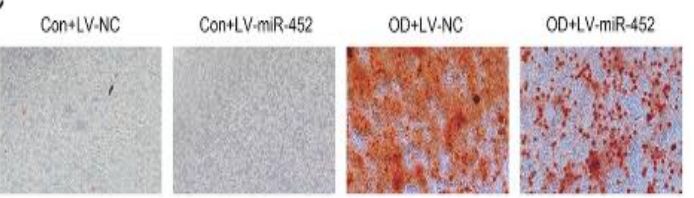

D

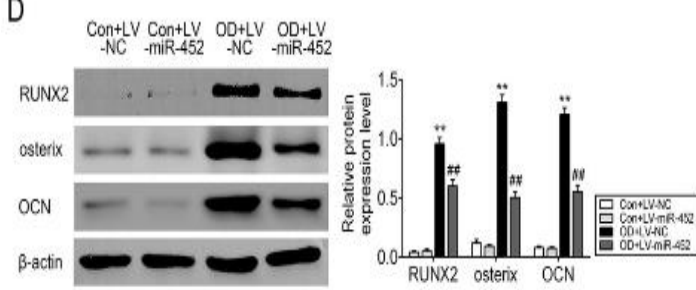

Figure 2. Overexpression of miR-452 inhibited OD of hPDLSCs. (A) qRT-PCR was used to measure miR452 expression in hPDLSCs. (B) ALP activity was measured in hPDLSCs. (C) Alizarin red staining was used to evaluate OD of hPDLSCs. (D) Western blot was used to measure protein expression of RUNX2, osterix, and OCN in hPDLSCs. ${ }^{* *} p<0.01$ vs. Con+LVNC group and ${ }^{\#} p<0.01$ vs. OD+ LV-NC group

qRT-PCR showed that miR-452 expression was increased by miR-452 mimics and decreased by miR-452 inhibitor in hPDLSCs (Figure $3 \mathrm{C}$ ). To validate whether miR-452 can modulate BMI1 expression, qRT-PCR was used to measure BMI1 expression. The results showed that BMI1 expression was decreased by miR-452 mimics, and was increased by miR-452 inhibitor in hPDLSCs (Figure $3 \mathrm{D}$ ). In addition, the western blot assay showed that BMI1 protein expression was decreased in hPDLSCs transfected with miR-452 mimics, and was increased in hPDLSCs transfected with the miR-452 inhibitor (Figure 3 E). These data showed that miR-452 could negatively regulate BMI1 expression in hPDLSCs.

\section{Expression of BMl1 during OD of hPDLSCs}

The mRNA and protein levels of BMI1 in hPDLSCs was higher at day7 and day 14 as compared with that at day0 $(p<0.05$; Figure 4$)$. These results indicated that the miR-452 expression was induced in hPDLSCs during OD, which may suggest a positive correlation between BMI1 expression and OD (Figure $4 \mathrm{~A}$ and $4 \mathrm{~B}$ ).
A

BM1-WT 5. AuaugacauaAcaggaAacagua..3

miR-452 3' AGUCAAAGGAGACGUUUGUCAA 5 '

BMI1-MUT 5'. AUAUGACAUAaCAGgUUUGUCAA...3'

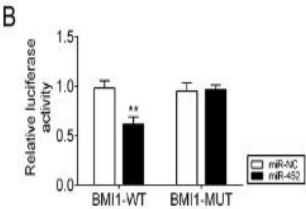

C

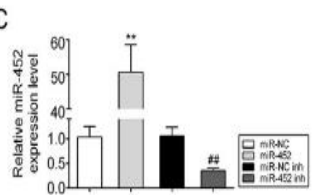

D

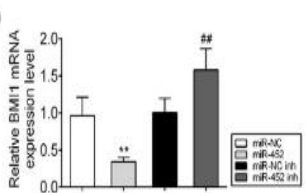

$E$
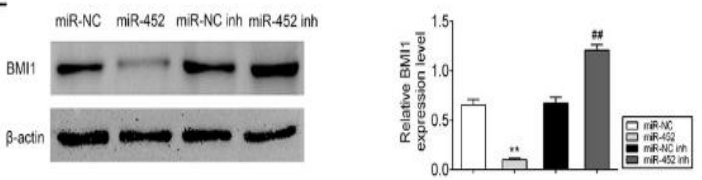

Figure 3: BMl1 was the direct target of miR-452. (A) This diagram showed binding sites between the miR452 and BMI1-WT sequences. BMI1-WT represents the entire 3'UTR sequence (625-631) of wild-type BMI1. BMI1-MUT represents the mutated 3'UTR sequence and could not bind to miR-452. (B) The luciferase reporter assay was performed to evaluate binding affinity of miR-452 to BMI1-WT/BMI1-MUT. (C and D) qRT-PCR was used to measure miR-452 and BMI1 mRNA expression levels in hPDLSCs. (E) Western blot was used to measure BMI1 protein expression in hPDLSCs. ${ }^{* *} p<0.01$ vs miR-NC, ${ }^{\# \#} p<$ $0.01 \mathrm{miR}-\mathrm{NC}$ inh
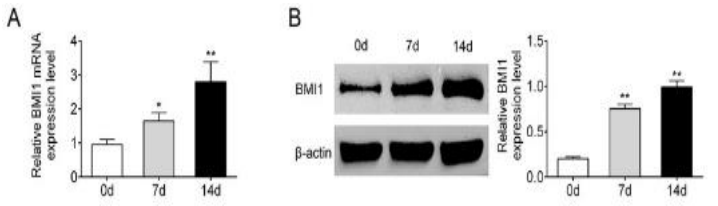

Figure 4. BMl1 expression in hPDLSCs during OD. (A) BMI1 mRNA expression was measured by qRTPCR. (B) BMl1 protein expression was measured by western blot. ${ }^{*} P<0.05$ and ${ }^{* *} p<0.01$ vs. day 0 , respectively

\section{Knockdown of BMI1 inhibited OD of hPDLSCs}

ShBMI1-transfected hPDLSCs were used to evaluate the functional effect of BMI1 during OD. The successful knockdown of BMI1 in hPDLSCs was confirmed (Figure $5 \mathrm{~A}$ ). Compared with the control group (shNC-transfected hPDLSCs), the ALP activity was inhibited in shBMl1-transfected hPDLSCs $(p<0.05$; Figure 5 B). In addition, Alizarin red staining showed that matrix mineralization was lower in shBMl1-transfected hPDLSCs when compared with shNCtransfected hPDLSCs $(p<0.05$; Figure $5 \mathrm{C})$. Furthermore, the expression of OD markers, such as RUNX2, osterix, and OCN, were downregulated in shBMl1-transfected hPDLSCs 
when compared with shNC-transfected hPDLSCs $(p<0.05$; Figure 5 D). These data showed that BMI1 promoted OD of hPDLSCs.
A

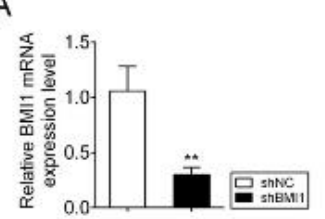

B

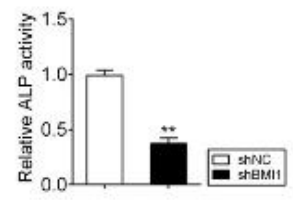

C
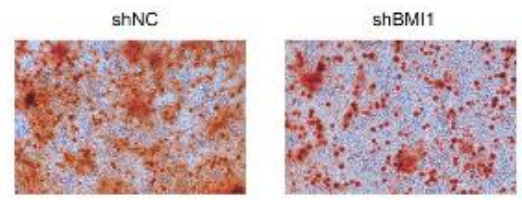

D

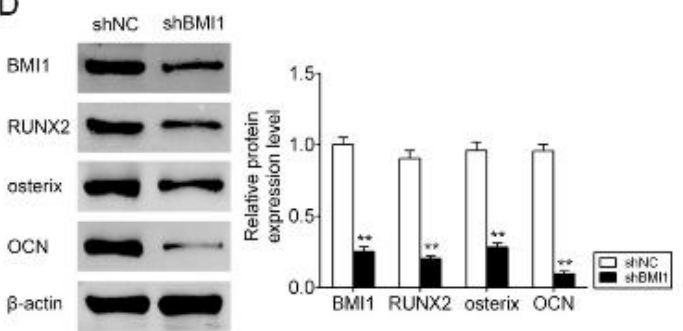

expression was decreased during $O D$ of hPDLSCs. Thus, miR-452 has potential as a biomarker for $\mathrm{OD}$, and overexpression of miR452 could inhibit OD. ALP activity and Alizarin red staining assays were used to evaluate progression of OD of hPDLSCs, and we found that miR-452 overexpression inhibited OD of hPDLSCs.

This study is the first evidence demonstrating that miR-452 regulates OD of hPDLSCs. In detail, the results showed that overexpression of miR-452 inhibits OD of hPDLSCs in vitro. Other studies have shown that miRNAs modulate OD via various molecular mechanisms. A previous study showed that miR-452-5p upregulated cell proliferation in colorectal cancer by interacting with CDKN1B [11], and miR-452 suppressed cell migration in breast cancer by targeting RAB11A [12]. Besides, in human cervical cancer, reduced miR-452 expression was related to vascular invasion, lymph node metastasis, poor tumor differentiation and short overall survival [13]. It has also been shown that miR-452 mediates tumor cell proliferation and invasion by targeting various genes via different signaling pathways [14]. However, the precise target of miR-452 in OD of hPDLSCs remains unclear.

To explore the molecular mechanism underlying the regulatory role of miR-452 in the progression of OD, the bioinformatics predicted miR-452 could bind directly to BMI1 3 'UTR-WT, which was also verified by luciferase reporter assay. In addition, miR-452 overexpression inhibited BMI1 expression in hPDLSCs, thus, BMI1 is a downstream target of miR-452. Furthermore, qRT-PCR, western blot, ALP activity, and Alizarin red staining assays showed that knockdown of BMI1 inhibited OD of hPDLSCs. Taken together, our data demonstrated that miR-452 suppresses OD of hPDLSCs by targeting BMI1. BMI1 is a polycomb-group protein and evidence have suggested that it plays a role in various cancers, including bladder cancer [15] and lung cancer [16]. It has also been shown that BMI 1 is regulated by numerous miRNAs that affect cell proliferation and differentiation. For example, overexpression of miR-27a and miR-155 inhibited cell proliferation and migration by directly targeting BMI1 [17]. Whether BMI1 is regulated by other miRNAs in the progression of OD needs further investigations.

multiple pathways. For example miR-24-3p promotes OD by targeting Smad5, and miR-214 inhibits OD of hPDLSCs by binding to ATF4. MiR-452, a widely known miRNA, attracted our attention due to its regulatory role in cell proliferation and differentiation in many human cancers. However, little is known about the role of miR-452 in the progression of OD. In this study, qRT-PCR assay revealed that miR-452

\section{CONCLUSION}

MiR-452 suppresses OD of hPDLSCs in vitro, and also miR-452 suppresses OD of hPDLSCs by targeting BMI1. Thus, miR-452 is a potential biomarker for detecting molecular pathogenesis 
of OD in hPDLSCs, and may ultimately lead to the development of novel therapies for periodontitis.

\section{DECLARATIONS}

\section{Conflict of interest}

No conflict of interest is associated with this work.

\section{Contribution of authors}

We declare that this work was done by the authors named in this article and all liabilities pertaining to claims relating to the content of this article will be borne by the authors. Tiantian Mao and Jun Li designed the study and supervised the data collection. Ruobing Peng analyzed and interpreted the data. Linhua Liu and Youjian Peng prepared the manuscript for publication and reviewed the draft of the manuscript. All authors have read and approved the manuscript.

\section{Open Access}

This is an Open Access article that uses a funding model which does not charge readers or their institutions for access and distributed under the terms of the Creative Commons Attribution License (http://creativecommons.org/licenses/by/ 4.0) and the Budapest Open Access Initiative (http://www.budapestopenaccessinitiative.org/rea d), which permit unrestricted use, distribution, and reproduction in any medium, provided the original work is properly credited.

\section{REFERENCES}

1. Di Benedetto A, Gigante I, Colucci S, Grano M. Periodontal disease: linking the primary inflammation to bone loss. Clin Dev Immunol 2013; 2013(503754.

2. Wang $L$, Shen $H$, Zheng $W$, Tang $L$, Yang $Z$, Gao $Y$, Yang $Q$, Wang $C$, Duan $Y$, Jin $Y$. Characterization of stem cells from alveolar periodontal ligament. Tissue Eng Part A 2011; 17(7-8): 1015-1026.

3. Dexheimer PJ, Cochella L. MicroRNAs: From Mechanism to Organism. Front Cell Dev Biol 2020; 8(409.

4. Wang $F$, Zhang Q-W, Fu X-H, Wang H-F, Liu Y-L. Expressions and clinic significance of miRNA-143, miRNA- 34A, miRNA-944, miRNA-101 and miRNA-218 in cervical cancer tissues. Trop J Pharm Res 2016; 15(7): 1387-1932.

5. Huang J, Zhao L, Xing L, Chen D. MicroRNA-204 regulates Runx2 protein expression and mesenchymal progenitor cell differentiation. Stem Cells 2010; 28(2): 357-364.

6. Luzi E, Marini F, Sala SC, Tognarini I, Galli G, Brandi ML. Osteogenic differentiation of human adipose tissuederived stem cells is modulated by the miR-26a targeting of the SMAD1 transcription factor. J Bone Miner Res 2008; 23(2): 287-295.

7. Wang $H, H u Z$, Wu J, Mei Y, Zhang Q, Zhang H, Miao D, Sun W. Sirt1 Promotes Osteogenic Differentiation and Increases Alveolar Bone Mass via Bmi1 Activation in Mice. J Bone Miner Res 2019; 34(6): 1169-1181.

8. Rao X, Huang $X$, Zhou Z, Lin X. An improvement of the $2^{\wedge}$ (-delta delta CT) method for quantitative real-time polymerase chain reaction data analysis. Biostat Bioinforma Biomath 2013; 3(3): 71-85.

9. Monsarrat $P$, Vergnes $J N$, Nabet $C$, Sixou $M$, Snead $M L$, Planat-Benard V, Casteilla $L$, Kemoun $P$. Concise review: mesenchymal stromal cells used for periodontal regeneration: a systematic review. Stem Cells Transl Med 2014; 3(6): 768-774.

10. Shimono M, Ishikawa $T$, Ishikawa $H$, Matsuzaki $H$, Hashimoto S, Muramatsu T, Shima K, Matsuzaka K, Inoue T. Regulatory mechanisms of periodontal regeneration. Microsc Res Tech 2003; 60(5): 491-502.

11. Gao L, Gan X N, Ye Z H, Liang L, Chen G, Qin X G. MiR452-5p may serve as an oncogene in colorectal cancer through targeting CDKN1B: a study based on bioinformatics analysis and dual-luciferase reporter assay. Int J Clin Exp Med 2019; 12(3): 2151-2166.

12. Li W, Li G, Fan Z, Liu T. Tumor-suppressive microRNA452 inhibits migration and invasion of breast cancer cells by directly targeting RAB11A. Oncol Lett 2017; 14(2): 2559-2565.

13. Dong $X$, Yang $Z$ L, Zhang J. Decreased miR-452 expression in human cervical cancer and its prognostic significance. European Journal of Gynaecological Oncology 2018; 39(1): 27-31.

14. Tang H, Zhang J, Yu Z, Ye L, Li K, Ding F, Feng X, Meng W. Mir-452-3p: A Potential Tumor Promoter That Targets the CPEB3/EGFR Axis in Human Hepatocellular Carcinoma. Technol Cancer Res Treat 2017; 16(6): 1136-1149.

15. Liang W, Zhu D, Cui X, Su J, Liu H, Han J, Zhao F, Xie W. Knockdown BMI1 expression inhibits proliferation and invasion in human bladder cancer T24 cells. Mol Cell Biochem 2013; 382(1-2): 283-291.

16. Meng $X$, Wang $Y$, Zheng $X$, Liu $C$, Su $B$, Nie $H$, Zhao $B$, Zhao $X$, Yang H. shRNA-mediated knockdown of Bmi-1 inhibit lung adenocarcinoma cell migration and metastasis. Lung Cancer 2012; 77(1): 24-30.

17. Li Y, Tian Z, Tan Y, Lian G, Chen S, Chen S, Li J, Li X, Huang $K$, Chen Y. Bmi-1-induced miR-27a and miR-155 promote tumor metastasis and chemoresistance by targeting RKIP in gastric cancer. Mol Cancer 2020; 19(1): 109. 